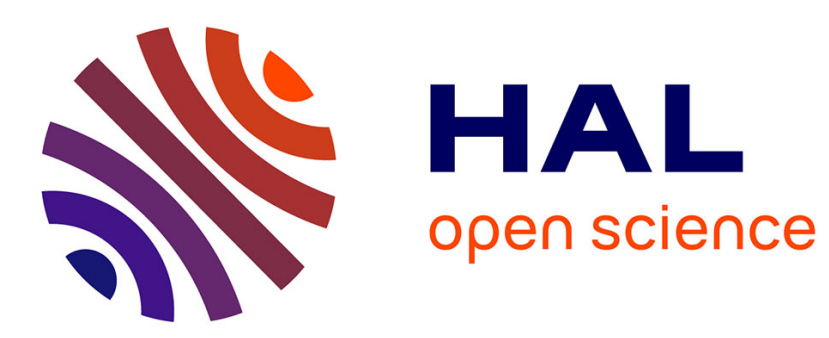

\title{
DU DÉSIR AU NIRVÃNA. LE DESTIN BOUDDHIQUE DE LA SEXUALITÉ
}

Elizabeth Kaluaratchige

\section{To cite this version:}

Elizabeth Kaluaratchige. DU DÉSIR AU NIRVÃNA. LE DESTIN BOUDDHIQUE DE LA SEXUALITÉ. Topique - Revue freudienne, 2016, 134, 10.3917/top.134.0021 . hal-01470903

\section{HAL Id: hal-01470903 https://hal.science/hal-01470903}

Submitted on 17 Feb 2017

HAL is a multi-disciplinary open access archive for the deposit and dissemination of scientific research documents, whether they are published or not. The documents may come from teaching and research institutions in France or abroad, or from public or private research centers.
L'archive ouverte pluridisciplinaire HAL, est destinée au dépôt et à la diffusion de documents scientifiques de niveau recherche, publiés ou non, émanant des établissements d'enseignement et de recherche français ou étrangers, des laboratoires publics ou privés. 


\title{
DU DÉSIR AU NIRVÃNA. LE DESTIN BOUDDHIQUE DE LA SEXUALITÉ
}

\author{
Elizabeth Kaluaratchige
}

L'Esprit du temps | « Topique »

2016/1 n $134 \mid$ pages 21 à 35

ISSN 0040-9375

ISBN 9782847953558

Article disponible en ligne à l'adresse :

http://www.cairn.info/revue-topique-2016-1-page-21.htm

\section{Pour citer cet article :}

Elizabeth Kaluaratchige, «Du désir au Nirvãna. Le destin bouddhique de la sexualité », Topique 2016/1 (n 134), p. 21-35.

DOI 10.3917/top.134.0021

Distribution électronique Cairn.info pour L'Esprit du temps.

(C) L'Esprit du temps. Tous droits réservés pour tous pays.

La reproduction ou représentation de cet article, notamment par photocopie, n'est autorisée que dans les limites des conditions générales d'utilisation du site ou, le cas échéant, des conditions générales de la licence souscrite par votre établissement. Toute autre reproduction ou représentation, en tout ou partie, sous quelque forme et de quelque manière que ce soit, est interdite sauf accord préalable et écrit de l'éditeur, en dehors des cas prévus par la législation en vigueur en France. Il est précisé que son stockage dans une base de données est également interdit. 


\title{
Du désir au Nirvãna. Le destin bouddhique de la sexualité
}

\author{
Elizabeth Kaluaratchige
}

«Comme un grand arbre bambou aux branches entrelacées, il est enchaîné à sa femme et à ses enfants ; comme la racine d'un bambou qui ne s'enlace pas, vous devrez cheminer tout seul, comme un rhinocéros » (Suttanipatha)

Le Bouddha donne des conseils à un homme : renoncer à la femme, renoncer aux enfants. Ces derniers l'empêchent d'atteindre le Nirvãna ${ }^{\mathbf{1}}$ : le but final du bouddhisme. Dans le Dhammapada, le texte le plus populaire contenant les paroles du Bouddha, le seizième chapitre traite des aspects liés à la sexualité tels que le désir, le plaisir, l'amour, l'attachement, la convoitise, les relations et les liens :

«Que personne n'aime rien. La mort du bien-aimé est insupportable. Ceux qui n'aiment rien et qui ne haïssent rien ne sont pas enchaînés. Du bien-aimé naît la souffrance. Du bien aimé naît la peur. Celui qui se libère du bien-aimé n'a pas de souffrance, d'où viendrait la peur? De l'amour naît la souffrance. De l'amour naît la peur. Pour celui qui se libère de l'amour, n'a pas de souffrance, d'où viendrait la peur? Du plaisir sensuel naît la souffrance. Du plaisir sensuel naît la peur. Celui qui se libère du plaisir sensuel, n'a pas de souffrance, d'où viendrait la peur? De la soif (le désir ou la convoitise) naît la souffrance. De la soif naît la peur. Pour celui qui se libère de la soif n'a pas de souffrance. D'où viendrait la peur?» (Dhammapada).

Dans le bouddhisme, la peur, le chagrin ou la souffrance, naissent non seulement par l'érotisme, le plaisir sensuel ou le désir, mais par les relations qu'ils

1. Le Nirvãna, mot sanskrit emprunté au Nibbãna de la langue pâli, langue canonique du bouddhisme, désigne étymologiquement «l'extinction d'une flamme » ou «une ex-spiration» : Nir $=$ sortir, Vãna $=$ le vent ou le souffle qui est associé au désir.

Topique, 2016, 134, 21-35. 
créent avec les objets de satisfaction, les choses et les personnes. Les relations entraînent l'homme vers le prolongement du cycle de vie, Samsara, qui génère la souffrance. Comment le noyau doctrinal du bouddhisme associe alors la sexualité, la vie et la souffrance ? Envisager une réponse à cette question exige une réflexion sur le thème de la « sexualité » dans le bouddhisme à travers deux aspects fondamentaux :

- les prescriptions bouddhiques dans les textes canoniques

- les enjeux pratiques qu'elles forgent dans l'organisation religieuse du bouddhisme.

Notre intérêt est ici d'étendre au bouddhisme, le dialogue que Freud a commencé autour de l'expérience religieuse. Il est susceptible d'éclairer la fonction de cette religion dans la culture, hors contexte monothéiste et polythéiste. Sans appliquer la psychanalyse au bouddhisme, visons plutôt le rapport entre le bouddhisme et la culture, pris par l'idéal «morale sexuelle civilisée des bouddhistes ». Plusieurs années de recherches sur le bouddhisme et la psychanalyse, nous permettent d'aborder le thème de la « sexualité » dans le bouddhisme, en tenant compte de l'usage des concepts désignant non seulement les «comportements observables », mais les phénomènes de la vie qui restent à l'arrière plan inconscient.

\section{LES CONCEPTS BOUDDHIQUES LIÉS À LA SEXUALITÉ}

Dans la perspective de cette étude, peut-on appliquer au bouddhisme le terme «sexualité », au sens scientia sexualis, le savoir du sexuel, né en Europe ? Le terme « sexe » ou « sexualité », linga ou lingika est plutôt une invention récente dans le contexte bouddhique. Il a ses racines remontant au terme lingam de la langue classique le pali, désignant le genre ou le sexe masculin, féminin ou neutre (purisalingam, itthilingam, napunsakalingam). Or la notion de sexualité ne pourrait être repérée par une étude de la pensée bouddhique sur le désir et son extinction, le Nirvãna. Le bouddhisme utilise plusieurs concepts pour aborder le thème de «la sexualité ». Doit-on apposer l'adjectif sexuel(le), substantivé, aux termes, notions, concepts et comportements qui désignent les théories forgées par les fondateurs du bouddhisme? Le terme Kama désigne le désir sensuel et ardent, la passion de l'amour. Il est devenu récemment dans la communauté cinghalaise, le terme qui désigne le plaisir ardent sexuel et il devient adjectif du terme désir Kamasha (le terme en cinghalais racine pali est Ajjhasaya). On sait que l'hindouisme accorde une place importante au Kama, qualifié de divinité faisant un des quatre buts de la vie, tandis que le bouddhisme conseille l'évitement des cinq plaisirs sensuels Pancakama, et propose le célibat aux moines. Les profanes doivent limiter la sexualité au lien conjugal. Le terme Raga est également utilisé pour décrire le plaisir. Ratiya serait plutôt l'attachement sexuel; Pema, l'amour sensuel ; Tanha, le désir, la convoitise ou la soif 
de satisfaire le plaisir par les liens aux objets. Par quel biais le bouddhisme considère-t-il que les liens d'amour et les relations avec l'objet ont un effet dévastateur pour l'homme?

\section{LE DÉTACHEMENT DES LIENS}

Dans la pensée bouddhique, tout phénomène, tout concept, n'existe qu'un seul et unique instant. Le « devenir» de toute chose est donc Anicca, qu' on pourrait traduire par un néologisme «l'impermanence » ou «éphémérité ». L'individu est un agglomérat constitué des agrégats : agrégat de la matière, des sensations, des perceptions, des formations mentales et de la conscience. Les agrégats, par le fait de changer sans interruption en autre chose, entraînent la souffrance, Dukkha. Le mental, Manas, est un autre organe attaché au corps comme l'œil ou l'oreille. L'agrégat des sensations contient six parties: sensations nées du contact de l'œil avec des formes visibles, de l'oreille avec les sons, du nez avec les odeurs, de la langue avec les saveurs, du corps avec les objets tangibles et de l'organe mental avec les objets mentaux, pensées ou idées (Rahula, 1961, 40). La conscience mentale est fondée sur sa base dans l'organe mental ; la pensée est son objet. Dans le bouddhisme qui annule l'existence d'une âme, Anatta, le corps est difficilement séparable du mental. Si le mental se détache des objets et des personnes, il arriverait à l'état de Nirvãna. Afin d'éliminer totalement la douleur ou la souffrance du monde, il faut donc en éliminer la racine principale, la «soif » ou le désir ou la convoitise, Tanha. Ce terme signifie également « la volonté d'être, d'exister, de re-exister, de devenir, de croître de plus en plus, d'accumuler sans cesse » (Rahula, 1961,52) ou la «volition mentale». Tant que cette «soif» existe, la personne se réincarne (renaît). Quand l'homme n'aura plus soif de plaisirs des sens, de relation au bien-aimé, aux enfants, à la richesse, à la puissance et même aux idées, aux opinions, aux théories, aux conceptions et aux croyances, il pourra atteindre le Nirvãna: «La cessation de la douleur» (Majjima Nikaya), «L'extinction du désir, de la haine et de l'illusion » (Samyutta Nikaya), «La cessation de la continuité et du devenir, bhavanirodha.» (Samyutta Nikaya). L'idéal nirvãnique se situe ainsi au niveau de la pratique ascétique des moines, Bhikkhu. Dans le Dhammapada, le Bouddha adresse aux moines: «Médite ô Bhikkhu, ne sois pas négligent. Ne laisse pas tes pensées tourbillonner sur les plaisirs des sens. » Contrairement au Petit Véhicule ${ }^{2}$ qui propose l'accès

2. Le Petit Véhicule est pratiqué au Sri Lanka depuis le $3^{\mathrm{e}}$ siècle avant J.-C., et il est répandu dans les pays tels que la Birmanie, la Thaïlande et le Laos. Malgré l'influence des cultes et pratiques ésotériques du Grand Véhicule, ce courant, dans son fondement théorique et pratique, reste plus proche de la doctrine des anciens, Théravada, notamment dans la communauté cinghalaise bouddhiste de Sri Lanka. 
au Nirvãna par la vie monastique, le Grand véhicule propose un « Sentier Rapide » aux profanes; le travail mental, les moyens corporels, les exercices de respiration, la méditation, les pratiques du yoga, les techniques magiques et ésotériques, des récitations des formules et des rituels... ${ }^{3}$.

Celui qui atteint le Nirvãna atteindra l'extinction complète, le Parinirvãna, après sa mort. Il pourra ainsi annuler définitivement la répétition des naissances et braver le travail du dieu de la mort, Mara. L'inspiration nirvãnique serait alors un héroïsme. Comme le note Freud, le héros est celui qui serait capable d'annuler la mort par bravoure. Le bouddhisme propose-t-il une solution compatible avec «notre inconscient inaccessible à la représentation de notre propre mort...? (Freud, 1915, 45). Comme nous l'avons montré ailleurs, le Bouddha représente le «surhumain» ou le «supra-humain» (Bopearachchi, 1995; Kaluaratchige, 2011b); il a vaincu le dieu de la mort ainsi que ses trois filles, Tanha (soif ou désir), Rati (plaisir ardent) et Ranga (danse); Lacan émet l'hypothèse qu'il y aurait le culte du Grand Homme dans le bouddhisme, et ce dernier serait resté à mi-route, en deçà du meurtre primitif du Grand Homme - le père de la horde (Lacan, 1986, 207). Peut-on dire que l'aspiration nirvãnique est adressée à l'héroïsme et au fantasme de «surhumain »?

\section{ARRÊTER DE COURIR DERRIÈRE LE DÉSIR-ILLUSION, MAYA}

Le bouddhisme explique que si la cause est éliminée, l'effet ou la production s'évanouit également. Si l'homme peut éliminer la cause de la souffrance, à travers la pratique du détachement, il peut éliminer la production du «je»ou le «ego», Aham. Il est plus facile ainsi de comprendre le concept de «désirillusion », Maya, du bouddhisme. Lacan, faisant référence au désir-illusion, ramène notre attention sur le désir qui n'est qu'un voile de Maïa, (Maya), le mirage (Lacan, 2001, 418). Le désir du sujet reste, au dernier terme, le désir de l'Autre ou le désir du corps de l'Autre (Lacan, 2004, 249), donc une illusion, Maya. Pour saisir le rapport entre le désir-illusion du bouddhisme et le désir dans la psychanalyse, il importe de se retourner vers Freud. L'aspiration au «bonheur absolu», selon les termes freudiens est le fantasme du «retour au giron maternel», Mutterliebphantaisie (Freud, Abraham, 1969, 352). Cependant, la castration ne permet pas d'y retourner. La perte de l'objet «sein » intervient en premier lieu, pour que l'enfant abandonne à regret le bonheur vécu à l'endroit

3. Le Grand Véhicule est né peu après le décès du Bouddha. Son véritable commencement se trouve dans le développement de l'école du Milieu en Inde, entre 150 et 250 après J.-C. En se qualifiant de Grand moyen de progression ou Grand Véhicule, Mahayana, elle surnomme l'école des anciens, Theravada, Petit Moyen de progression ou Petit Véhicule, Hinayana. Le Grand Véhicule chemine jusqu'à la Chine, au Japon et à la Corée. 
de la mère. Freud illustre comment le sein devient l'organe de satisfaction pulsionnelle qui appartient à l'enfant et à la mère (Freud, 1930, 8), et que l'enfant perd retrouvant d'autres objets qui chutent également plus tard. Lacan par son invention du concept d'objet $a$, introduit la liste des objets que l'enfant perd : le sein, l'excrément, le regard, la voix, le phallus imaginaire... Ils sont «inatteignables » dans le réel, sauf que le sujet peut continuer à désirer dans le symbolique. Le concept « soif» insatiable, Tanha, du bouddhisme désigne, nous semble-t-il, cette tentation interminable du sujet à atteindre ce qui est inatteignable.

Le bouddhisme propose alors de ne pas prendre en compte ce ratage ou le mirage du désir. Lacan explique ce phénomène par l'objet $a$ qui n'est qu'une cible. On ne l'atteint que «d'un tir qui le rate» (Lacan, 2001, 310). Il n'appartient pas au registre spéculaire, et il n'a pas d'altérité, c'est ainsi qu'il demeure l'objet de l'angoisse (Lacan, 2004, 50-60). Si on suit ce chemin du désir-illusion, on peut supposer que le bouddhisme, par ses conseils d'éliminer la soif, propose une solution à la racine même de l'angoisse: l'élimination de l'illusion, arrêter de courir derrière le mirage du désir de l'Autre. Dans les Séminaires Les paupières de Bouddha et La bouche et l'œil, Lacan avance l'hypothèse que les postulats bouddhiques proposent une fermeture aux fonctions de la cause. Sans aucune mention d'un «dieu créateur de l'univers », le bouddhisme s'appuie sur la diaphragmatisation de la cause première. Selon Lacan, le point d'angoisse qui se situe à l'endroit du sein, n'est pas le désir qui se situe à l'endroit de l'enfant. L'image du Bouddha avec ses yeux baissés montre qu'il prend le point d'angoisse, tout entier à sa charge, et suspend, annule apparemment «le mystère de la castration » (Lacan, 2004, 247-279). L'aspiration à l'état de plénitude est d'aller vers l'état sans castration ni désir; la sortie de l'engrenage de la vie libidinale. Peut-on dire que le bouddhisme comme «toute formation humaine a pour essence, et non pour accident, de refréner la jouissance»? La Chose nue de la jouissance est bien cachée, selon Lacan, par «le prisme de la religion, le frein de la jouissance» (Lacan, 2001, 364). Le Nirvãna serait-il la solution la plus radicale pour freiner la jouissance?

\section{SUBLIMATION ET AU-DELÀ DU PRINCIPE DE PLAISIR}

Malgré la sublimation, certaines techniques inventées par les sages bouddhistes conviennent au fantasme de l'homme voulant atteindre, comme le note Freud d'après Schiller, «se réjouit dans la lumière rose, car en-dessous, c'est l'épouvante» (Freud, 1930, 15), de se retourner vers «le giron maternel», ou à la «masse des sensations » (Freud, 1930, 8), sans pour autant sortir du filet de la culture. Lou Andreas-Salomé se demande si la sublimation n'est pas un équilibre entre le sexuel et le spirituel, fondée sur «une nostalgie» de l'objet 
premier (Freud, Andreas-Salomé, 1970, 16). En faisant référence à Freud, elle explique que toute sexualité a une nostalgie. Il peut exister des personnes qui jouissent dans le «giron maternel en une identité avec l'univers qui l'environne » (Freud, Andreas-Salomé, 1970, 35). Peut-on dire que le sujet qui s'arrête de courir derrière le désir de l'Autre, essaye de réaliser au contraire le désir de l'Autre? Que se passe-t-il alors au niveau de la chaîne des signifiants dans le langage? L'arrêt des liens et des relations peut être alors qualifié de «gel des signifiants » (Kaluaratchige, 2013, 113). Les personnes qui répètent intensivement ces pratiques témoignent de leur souhait de réaliser la jouissance par leur sensation de «dissolution » dans l'univers. Cependant, elles sont conscientes de leurs pratiques. On peut supposer que le pratiquant appelle l'Autre « comme il veut» et «quand il veut»; il répètera le détachement mais l'investissement se fait par la sublimation. Il pourra alors créer une «réalité spirituelle », selon le choix du moment de pratique : heure, jour, période de l'année... Il semble vivre un moment extatique de détachement des liens, où le corps de sensations changerait par l' «ivresse spirituelle» (Mijolla-Mellor, 2004). On sait que pendant «La Renaissance orientale», les savants, les artistes occidentaux tels que Michelet, Schopenhauer, Nietzsche, Wagner restent marqués par la pensée bouddhique; ils ont vécu les moments d'ivresse spirituelle, certains sont devenus les pratiquants des techniques introduites par le bouddhisme (Schwab, 1950). Les pratiquants assidus risquent également de cheminer vers la «dépendance spirituelle» (Kaluaratchige, 2008c). Freud se méfie de tout excès de la Schwärmerei, qui renvoie aux tentations d'exaltation et d'illumination qui visent «le dépassement des limites du gain de plaisir imposées par le principe de réalité » (Kaluaratchige, 2014, 138). L'homme qui se concentre sur le seul acte de respiration fait un retour momentané vers le repos qui ressemble à une plénitude sourde et muette (Kaluaratchige, 2013,112). Un tel pratiquant semble dire que l'origine de toute chose est elle-même muette (Assoun, 1982, 11). Lacan retravaille le concept de principe de Nirvãna de Freud pour expliquer que le principe de plaisir vise au fond, Au-delà de principe de plaisir, la mort et sa dissolution : l'état avant l'apparition de la vie (Lacan, 1978, 102). Freud, au début de la découverte du principe de Nirvãna, retravaille les hypothèses de Weismann qui distingue une moitié mortelle et une moitié immortelle de la substance vivante (Freud, 1920). La moitié mortelle est le corps au sens étroit, le soma soumis à la mort naturelle. La moitié immortelle contient des cellules germinales qui sont capables de former un nouvel individu, de s'entourer d'un nouveau soma. Freud montre que chez les humains, la pulsion sexuelle représente cette tendance de «l'éternel» qui pousse l'homme à désirer, à construire, à reproduire et à se développer dans la civilisation. Suivant Schopenhauer, Freud ajoute que «la mort est bien le propre résultat » de la vie (Freud, 1920, 107), mais les hommes veulent toujours prolonger le circuit de la vie, avant d'aller vite vers la mort. En revanche, le bouddhisme propose le détachement du désir, du lien et de la 
reproduction, ce qui annule l'écoulement de la vie d'amour, la souffrance et la mort. L. Andreas-Salomé semble avoir apprécié cet aspect comme la sérénité face à la mort (Andreas-Salomé, 1983, 54).

Le corps en position immobile d'un méditant assidu nous prouve-t-il la libération de l'homme assiégé par la souffrance? Ou bien, représente-t-il la pétrification de l'homme face à la souffrance ou à l'angoisse insoutenable ? La métaphore de "pierre immobile » à l'appui, Lacan fait une équation par une série d'arguments, pour dire que l'homme arrête de rouler les pierres en les dressant. L'architecture elle-même devient ainsi la « présentification » de la douleur (Lacan, 1986, 74). Daphné se transforme en arbre sous la pression d'une douleur à laquelle elle ne peut plus échapper. Le devenir « immobile » n'évoquerait-il pas ainsi le suicide, mais la fin du désir, la mort du sujet du désir? Autrement dit, le principe de Nirvãna ne désigne pas la mort des êtres vivants, mais la fin du vécu humain, de l'échange humain ou de l'intersubjectivité (Lacan, 1978, 103). Contrairement à Hans Im Glück, mentionnée par Freud, (Waldinger, 1941, 4 ; Molnar, 1992, XXIV-XXV) qui continuait son circuit symbolique, un homme qui choisit le «Sentier Rapide», le chemin de la délivrance, se précipiterait vers la mort de la libido; le Bonheur (Glück) est selon Freud, la réalisation retardée d'un désir préhistorique. Il anticiperait la fin libidinale (Freud, S. Ferenczi, 1992, 321). Le circuit de désir est très long et exige beaucoup d'énergie libidinale : «S'il y ramenait par les voies les plus courtes, le problème serait résolu » ajoute Lacan. Il s'agit d'aller au-delà du plaisir qui, par définition, tend à sa fin. Autrement dit, le principe du plaisir explique que le plaisir cesse (Lacan, 1978, 107). Pour ne pas tuer le plaisir, il faut retenir la tension pulsionnelle. Lacan ajoute ironiquement : «Il faut retenir son foutre, pour être bien. Le bouddhisme, lui est l'exemple trivial par son renoncement à la pensée elle-même. Ce qu'il y a de mieux dans le bouddhisme, c'est le zen, et le zen, ça consiste à ça - à te répondre par un aboiement, mon petit ami. C'est ce qu'il y de mieux quand on veut naturellement sortir de cette affaire infernale, comme disait Freud.» (Lacan, 1975, 146). Sans décharger la tension pulsionnelle par l'objet, garder la tension à un niveau élevé permet-t-il de réaliser la jouissance de l'Autre?

\section{LA QUESTION DU FÉMININ, LA FEMME ET L'ASPIRATION NIRVÃ- NIQUE}

À partir d'une réflexion sur l'aspiration nirvãnique, Lacan ré-interroge la jouissance féminine et les phénomènes mystiques. La statue du bodhisattva (les intercesseurs du Bouddha) japonais Kwan yin, est une divinité plutôt féminine ou une divinité au sexe psychologiquement indéterminable (Lacan, 2004, 261). Lacan rappelle que son nom venant du Sanskrit Avalokiteshvara est répandu dans la sphère mondaine qui pratique le yoga. La forme féminine et le sexe 
indéterminé du bodhisattva, nous permettent de la présenter comme la figure de la Chose impossible, «l'archaïque du maternel». On sait que le débat entre Freud et Romain Rolland (Kaluaratchige, 2008b) se centre autour du sentiment océanique associé à l'éternité et à la mer. Freud qualifie ce moment du retour au moi-plaisir primitif, l'état avant l'objet séparé (Freud, 1930, 8). Dans la tradition indienne, un Grand Homme, tel que le Bouddha, est caractérisé par l'organe du sexe masculin atrophié. Peut-on dire qu'il est associé au clitoris ? L'aspirant au Nirvãna obéit-t-il au destin d'un fils féminisé ou châtré par la jouissance féminine ?Que peut-on dire alors du renoncement à l'amour sexuel de la femme ? Le Bouddha était très réticent à accorder le droit à la femme de devenir moniale. Il annonce que c'est un danger pour le système monastique car la femme a des difficultés réelles et psychologiques de vivre une vie solitaire, «sans foyer » et sans liens. Selon le bouddhisme, la femme n'ayant pas le droit de devenir un Bouddha pendant sa vie actuelle doit aspirer avant tout, à devenir un homme (Bopearachchi, 1994, 208-211, Wijayaratne, 1991, 21-24).

\section{LE KARMA, LA CULPABILITÉ ET LE SURMOI TYRANNIQUE}

Le Nirvãna n'est atteint que par le détachement des désirs/plaisirs, des liens/relations. La légende du Bouddha raconte que le bodhisattva (l'intercesseurs du Bouddha) avance progressivement pendant 550 naissances. Dans sa $549^{\mathrm{e}}$ vie, il se sépare de sa femme, et offre ses enfants comme serviteurs d'un homme riche. Dans sa dernière naissance, il quitte le foyer, et abandonne sa femme et son enfant qui venait de naître. Il nomme son enfant Rahula, désignant «la cause de l'amour ». A. Foucher écrit: « Notre âge du monde a vu un Bouddha éclore, le terme de son évolution multimillénaire.» (Foucher, 1987, 179). Les humbles humains peuvent-ils espérer mieux que le Bouddha ? Devant la difficulté de devenir Bouddha, certains villageois vont jusqu'à dire «Une fois vieux, même le démon peut se faire moine», «Quand on ne pourra plus bouger les bras et les jambes, on se rappellera les paroles du Bouddha », «Laissons la place aux gens pressés de devenir Bouddha».

Le bouddhisme fait entendre que ceux qui n'ont pas pu éradiquer les liens, naissent chargés des effets de leurs propres actes, Karma, commis pendant leurs vies antérieures. Les effets karmiques sont déterminés selon les qualités des actes, bons ou mauvais. Seuls quelques actes mauvais porteront leurs fruits pendant la vie actuelle. Les conditions socio-économiques, la beauté, la santé au même titre que le succès dans l'amour, sont les résultats des actes commis par la personne (Bopearachchi, 1994, 174). Max Weber montre que la doctrine du Karma a donné une réponse satisfaisante à la société des profanes (Weber, 1958, 275). Pour R. Gombrich, la loi karmique reste au centre du bouddhisme comme moyen de traiter l'omnipotence ou «l'ominibénévolence» attribuée au Dieu 
monothéiste et résout «la question de la théodicée » (Gombrich, 1971, 144). Le bouddhiste qui ne rencontre pas de sentiment de culpabilité face au Dieu tout puissant, souffre autrement du sentiment d'avoir commis un acte mauvais luimême ou par son propre « ancestralité ». Peut-on voir dans la doctrine du Karma, la culpabilité originaire portée par la phylogenèse mais oubliée par le sujet dans l'ontogenèse ? Quelle forme prend la culpabilité dans la communauté des bouddhistes ? Freud traite de la culpabilité à partir des religions monothéistes telles que le judaïsme et le christianisme. Dans le cas du bouddhisme, la culpabilité a un lien étroit avec la doctrine du Karma. Le terme Karma a une connotation presque négative chez les profanes; il devient Karume dans la langue cinghalaise, désignant le destin impitoyable de supporter les préjudices, les injustices, les maladies physiques ou psychiques, les échecs... En dépit de la méconnaissance des vies antérieures, l'homme sait que les conséquences des actes « intentionnellement commis » sont inévitables. Le karume nous renvoie au destin impitoyable, le surmoi tyrannique, que Freud compare aux sorcières auprès du berceau, à la puissance des divinités parentales et à la Mö̈ra grecque, la déesse terrible et incontrôlable (Freud, 1924) sachant que le surmoi remonte au surmoi parental et jusqu'à l'ancêtre originaire. Ajoutons alors que le Karma n'est qu'un «mythe endopsychique » (...), le destin qui frappe le sujet dans le réel qui ne demande qu'à lui obéir (Assoun, 1999, 66).

\section{LA VIE D'AMOUR DES PROFANES, GIHIET L'IDÉAL «MONOGAMIE »}

Quelle serait alors la vie d'amour et du sexuel que peuvent espérer les profanes? Ils sont déjà nés des mauvais résultats karmiques. Les profanes sont des personnes qui ne peuvent pas éviter les liens d'amour ni les rapports sexuels pendant cette vie même. Quelles prescriptions leur propose le bouddhisme? La définition de la réincarnation selon les actes, Karma, suppose que, attachés par les liens invisibles maintenus dans le cycle de vie, Samsara, les femmes ou les hommes seront attirés les un(e)s par les autres, et se réuniront par le lien d'amour ou par le lien de mariage. Face à la difficulté inévitable de se libérer des relations, l'homme ne peut que respecter un «attachement idéal», une morale minimale : rester fidèle à un seul homme ou à une seule femme, telle qu'elle est prescrite par le Bouddha lui-même. Comme nous avons traité ailleurs, la monogamie est l'idéal de la sexualité pour les profanes, dont le modèle est la vie antérieure du Bouddha lui-même et son épouse Yasodhara qui étaient fidèles pendant 550 naissances dans le cycle de vie, Samsara (Bopearachchi, 1991). Le fait que le bodhisattva se trouvait dans la situation d'un profane prouve qu'il a vécu une vie d'amour et sexuelle. Dans les textes canoniques, on lit l'histoire des parents de Nakula illustrant les conseils du Bouddha adressés aux couples : «Vous qui vivez dans le foyer; si la femme et l'homme désirent être ensemble 
pendant cette vie et pendant la vie qui viendra, ils doivent être en harmonie dans la même croyance, la même vertu, la même générosité, la même intelligence » (Anguttara Nikaya). Les amoureux, après une offrande au temple, ou après un acte bon, font un vœu pour se réunir dans la vie prochaine. Au XXI ${ }^{\mathrm{e}}$ siècle, il n'est pas rare de rencontrer les couples qui souhaitent voyager ensemble pendant le cycle de réincarnations, Samsara. Une chanson d'amour en témoigne:

«Pendant mille ères, en pratiquant les vertus,

Parmi les obstacles, les souffrances et les douleurs sans fin, j'aspirais à être avec toi.

En voyant les signes de ton chemin.

D'une naissance à une autre, je suis venue te chercher.

En faisant les bagages du désir pour un voyage sans fin.

Courant derrière le mirage en sanglotant

Pour jouer du violon de la vie à côté de toi, je suis venue te chercher.

J'ai traversé les sept océans, j'ai couru entre les sept grandes montagnes

J'ai marché sous la lumière des étoiles, je suis venue te chercher ${ }^{4}$.»

La fidélité serait relayée par la modération dans la vie sensuelle. Les bouddhistes disent «On mange la viande sans en mettre autour du cou». Il valait mieux aimer une seule femme ou un seul homme, que d'être attaché à plusieurs ! Le bouddhisme aurait fait le même travail autrement, que «l'éthique protestante », pour reprendre le pasteur Pfister : «On a retiré aux relations sexuelles l'odieux de l'impureté » (Freud, Pasteur Pfister, 46). Un minimum de la « morale sexuelle bouddhiste » est exigée des couples qui mènent une vie séculière, observer Cinq Préceptes (Pancasila) : s'abstenir de détruire la vie, de prendre les objets qui ne sont pas donnés, de commettre l'adultère, de mentir, de boire des boissons enivrantes. Pour Max Weber c'est «l'éthique incomplète pour les plus faibles » (insufficiency ethic of the weak) (Weber, 1958, 275). Suivant le niveau de formation des individus, ces préceptes deviennent plus exigeants et complexes : 5 divisions et 8 divisions pour les profanes, et 10 divisions pour un «état plus élevé », pour les nonnes et les moines. Certains pratiquants bouddhistes essayent de pratiquer tous les jours, les cinq préceptes, malgré les obstacles de «la vie de désir». Respecter les préceptes ne représente aucune obligation, et laisse la place au bon vouloir et à l'exigence de chaque individu. Elle comporte également différentes modalités, une fois par jour, par semaine ou par mois (lunaire). 3 préceptes sont ajoutés aux 5 préceptes mentionnés plus haut pour en faire 8 :

- Ne pas prendre de repas à une heure non prescrite par les préceptes

- S'abstenir de danser, de chanter, de jouer de la musique, de regarder des spectacles malséants, d'utiliser des guirlandes, des parfums, de l'eau parfumée, de l'huile parfumée, des bijoux, de dormir dans des lits trop confortables

4. Chantée par Nanda Malini. 
- Éviter toute relation sexuelle.

Le jour de la pleine lune, habillés de blanc, les couples vont au temple pour pratiquer les 8 préceptes et s'abstiennent de rapports sexuels pendant au moins vingt-quatre heures. Reprenons ici les thèses de l'école anglo-saxonne. Pour Southwold, dans cette religion, il y a deux bouddhismes : «le bouddhisme villageois » et «le vrai bouddhisme» (Southwold, 1978, 137-151). Spiro voit un bouddhisme nirvanique et un bouddhisme karmatique. Il sépare ainsi les deux pratiques pourtant entremêlées dans une religion qui s'appuie sur une communauté de moines visant à atteindre le Nirvãna dans une période courte, et une communauté des profanes accordant une priorité aux actes bons afin de se réincarner dans une vie ultérieure plus élevée avec leur bien-aimé, le Nirvãna étant difficile à atteindre pour un profane (Spiro, 1971, 16-17). On sait que les actes bons tels que les dons, Dana, procure des mérites à l'individu ou transférables aux autres (Bopearachchi, 1992). Cependant, on observe, dans le Petit Véhicule, que les moines et les laïcs vivent un seul bouddhisme dans des interactions indispensables à la cohérence (Bopearachchi, 1994).

\section{LES PRÉCEPTES ET LE SURMOI CULTUREL}

En dépit de l'absence divine, les préceptes représentent la loi symbolique. Le dhamma, la loi bouddhique serait le signifiant père placé à l'endroit du signifiant flottant pour faire fonctionner la culture (Lacan, 2001, 277 ; Lévi-Strauss, 1950, XLIX-L, Kaluaratchige, 2011, 166-167). Les préceptes bouddhiques, au même titre que les dix commandements sont-ils là pour que le sujet prenne distance avec la Chose ? (Lacan, 1986, 87-102). La communauté bouddhiste serait alors constituée de degrés d'exigence différents par rapport à la vie d'amour et sexuelle.

Les préceptes bouddhiques qui sont établis sur une échelle de 5 à 10 , peuvent-ils être assimilés aux niveaux de gourmandise et d'exigence du surmoi culturel (Kultur-Überich) ? Comme le note Freud, le surmoi culturel «se soucie très peu, dans la sévérité de ses commandements et interdits, et ne prend pas suffisamment en compte les résistances contre leur observance, la force pulsionnelle du ça, et les difficultés du monde réel » (Freud, 1930, 86). On sait que «plus l'observance du précepte est difficile, plus elle est méritoire », la promesse étant «un au-delà meilleur». Comme le bouddhisme qui propose des étapes, peut-on dire que l'homme doit obéir au surmoi culturel selon ses capacités? Celui qui observe les cinq préceptes tous les jours, aura une tendance à aller vers les 8 préceptes, ainsi de suite. On sait que «après chaque satisfaction pulsionnelle empêchée pourrait avoir pour conséquence un accroissement du sentiment de culpabilité » (Freud, 1930,81-87). Les bouddhistes cinghalais ont tendance à dire «nous sommes des Karumakkarayo», autrement dit ceux qui 
ont un «sentiment d'avoir commis les actes mauvais pendant les vies antérieures».

Comme l'écrit Lacan, nos désirs doivent être éveillés dans leurs rapports à la Loi. Dans le bouddhisme qui exige le détachement complet du désir, du plaisir, de l'amour et du sexuel, l'objet de la sublimation, l'objet (de la sublimation) peut-être élevé à la dignité de la Chose (Lacan, 1986, 134). C'est la raison pour laquelle le bouddhisme n'imposerait pas au couple une loi véritable contre la séparation. L'idéal final étant le détachement du plaisir des sens, l'homme ou la femme a le droit de quitter la famille et de renoncer à la vie amoureuse. L'union de la femme et de l'homme n'étant pas une institution fondée sur le pouvoir divin, le bouddhisme facilitait toujours la séparation des hommes et des femmes et leur remariage sous certaines conditions (Knox, 1983, 174). Les raisons seront, la plupart de temps, considérées et discutées par les familles des partenaires selon le contexte moral, social et économique.

\section{LA PIÉTÉ FILIALE CONTRE LA FAMILLE EN EXTINCTION?}

On sait que comme le note Lacan, il n'y a que le désir qui maintient l'espèce, par le renouvellement de désir (Lacan, 1966, 812). Peut-on dire alors que le bouddhisme au sens «absolu» serait la fin de l'espèce ? En effet, dans les Canons bouddhiques, le Bouddha lui-même est accusé d'avoir proposé la fin de la lignée : «Le Samana Gotama encourage les hommes à ne pas avoir de fils. Le Samana Gotama rend les femmes veuves. Le Samana Gotama conduit les familles à l'extinction » (Vinaya Pitaka). J. Brosse, un occidental devenu bouddhiste à son arrivée en Chine, émet l'hypothèse que le monachisme du bouddhisme qui déconseille la descendance a posé des problèmes à l'empire chinois (J. Brosse, 2003, 34). Contrairement au bouddhisme du Grand Véhicule qui propose aux profanes les pratiques rigoureuses et le «Sentier Rapide», le bouddhisme du Petit Véhicule n'encourage pas les profanes à utiliser les techniques ésotériques, et mentales et surtout ne propose pas l'aspiration à l'état Nirvãnique ou à la «bouddhéité ». La peur de l'extinction de l'espèce, l'arrêt de la lignée, a fort probablement introduit la morale bouddhique de «la piété filiale» ou «la piété familiale»? L'homme doit servir la famille, les enfants et les parents. Peut-on dire qu'il s'agit d'une solution proposée par formation réactionnelle au détachement proposé par le bouddhisme? Les bouddhistes ont-il trouvé un moyen, par la sublimation, de résoudre les complexes parentaux ou les enjeux incestueux ? La communauté bouddhiste cinghalaise propose de vénérer littéralement les parents, de se prosterner à leurs pieds. Les bouddhistes ont également «bouddéisé » la mère : «le bouddha de la maison ». On peut compter des milliers de chansons sur la vénération de la mère, « la source d'amour », « la protectrice», «la pieuse » « la sainte», dans la communauté bouddhiste cin- 
ghalaise. «Le Bouddha de la maison» doit protéger la famille; c'est elle qui représenterait l'attachement et l'amour. Devant le danger d'abandon de la famille, des parents, des femmes, des enfants, ont-ils inventé une morale forte autour de l'amour pour les parents notamment pour la mère, pour tenir le sujet dans le filet de l'institution familiale?

\section{Elizabeth KALUARATCHIGE kaluaratch@outlook.fr}

\section{BIBLIOGRAPHIE}

ANDREAS-SAlomÉ L., Lettre ouverte à Freud (1931), Paris, Lieu commun, 1983.

Assoun P.-L.,

«Le sujet du destin. Figures freudiennes du destin», in Freud et le destin, Logos $\diamond$ Anankè, $\mathrm{N}^{\circ} 2 / 3,1999,2000, \mathrm{p} .105-126$.

«L'archaïque chez Freud », «Entre Logos et Anankè », in Nouvelle Revue de Psychanalyse, Archaïque, Paris, Gallimard, 1982, p. 11-44.

BOPEARACHCHi E.,

«Amour conjugal dans le bouddhisme cinghalais », in Cahier de l’Asie du Sud-Est, $N^{\circ} 29$ 30, INALCO, 1991, p. 93-110.

«Le Bouddha et la notion de «sauveur »», in Le Sacré en Eurasie, $N^{\circ} 5$, Pub. Avec le concours de la ville de Paris, 1995.

«Le don dans la littérature traditionnelle singhalaise», in Donner et recevoir, CREOPS, PUPS, 1992, p.59-69.

L'éducation bouddhique dans la société traditionnelle au Sri Lanka, Paris, L'Harmattan, 1994.

Brosse J., L'univers du zen, Histoire, spiritualité et civilisation, Paris, A. Michel, 2003.

Foucher A., La vie du Bouddha, Paris, 1987.

FREUD S.,

Le malaise dans la culture(1930), Paris, PUF, Quadrige, 2000.

«Considérations actuelles sur la guerre et sur la mort » (1915); «Au-delà du principe de plaisir» (1920), in Essais de Psychanalyse, Paris, PBP, 2001.

«Le problème économique du masochisme» (1924), in Névrose, psychose et perversion, Paris, PUF, 1999.

Freud S., Abraham K., Correspondance (1907-1926), Paris, Gallimard, 1969.

Freud S., ANdréAS SAlomé L., Correspondance avec Sigmund Freud, Paris, Gallimard, 1970.

Freud S., FerencZi S., Correspondance Tome II (1914-1919), Paris, Calmann-Lévy, 1992. Gombrich R.F., Precept and Practice, Oxford, 1971.

KALUARATCHIGE E., «La musique océanique : Romain Rolland entre Beethoven et Wagner», in Topique, Revue Freudienne, ${ }^{\circ} 128,214$, p. 135-149. 
«Le principe de Nirvãna et la jouissance», in Le Courrier des addictions, 2008c, p.24-26. «Écouter respirer...l'irrespirable: geler le «flot vocalique»», in La voix sur les braises, Rapport du Colloque, Solipsy, 2012, p.103-117.

«Freud versus Jung: Analyse versus Synthèse», Recherches en Psychanalyse [En ligne],। 2011. URL: http://recherchespsychanalyse.revues.org/2139.

«Les fonctions du père dans le bouddhisme, du lien à l'identification ", in L'Inde de la psychanalyse, dir. L. Boni, Campagne Première, Paris, 2011, p.165-180.

«Protection d'un père exalté ou refuge dans le sentiment océanique. Source et origine des besoins religieux à travers le débat Freud et Roman Rolland sur les religions indiennes », in Psychologie Clinique, $\mathrm{N}^{\circ} 26,2008 \mathrm{~b}$, Paris, L’Harmattan, p.55-71.

Le principe de Nirvãna et la jouissance dans la clinique du sentiment océanique, Les formes de la jouissance dans l'auto thérapie corporelle et spirituelle du sujet bouddhiste occidental moderne, Thèse de Doctorat, Université Paris 7, 2008a.

Knox R., Relation de l'île de Ceylan, La Découverte, Paris, 1983.

LÉvi-Strauss C., «Introduction à l'œuvre de M. Mauss», in M. Mauss, Sociologie et Anthropologie, Paris, PUF, 1950.

LACAN J.,

Autres écrits, Paris, Seuil, 2001.

Le Séminaire, L'angoisse, Livre X (1962-1963), Paris, Seuil, 2004.

Le Séminaire, Encore, Paris, Seuil, Points Essais, 1975.

Le Séminaire, Le moi dans la théorie de Freud et dans la technique de la psychanalyse, Paris, Seuil, 1978.

Le Séminaire, L'éthique de la psychanalyse (1959-1960), Paris, Seuil, 1986.

Mijolla-Mellor S. de, «Les ivresse sacrées », in Topique, L'esprit du temps, 2004, p. 3552.

Molnar M., Sigmund Freud, Chronique la plus brève, Carnet intimes 1929-1939, Albin Michel, 1992.

RahUla W., L'enseignement du bouddha, Paris, Seuil, Folio Sagesse, 1961.

Schwab R., La Renaissance Orientale, Paris, Payot, 1950.

Southwold M., «True Buddhism and Village Bouddhism », in Journal of Asiatic Society, 1978.

SPIRO M. E., Buddhism and Society, London, 1971.

WEBER M., Religion of India, trad. Angl., Newyork, 1958.

WALDINGER E., My Uncle Sigmund Freud, Books Abroad, vol. 15, №1, 1941.

WiJAYARATNA M., Les moniales bouddhistes, naissance et développement du monachisme féminin, Paris, cerf, 1991.

Textes Pali et sanskrit

Majjhima-nikaya, PTS. London,

Samyutta Nikaya, éd. L. Feer, PTS, London.

Dhammapada, trad. ang. F. M. Müller, Oxford, 1898.

Suttanipata, trad. Ang., V. Fausböll, Delhi, 1881.

Vinaya Pitaka, Vinaya Texts, I. B. Horner, Oxford, 1957. 
LE DESTIN BOUDDHIQUE DE LA SEXUALITÉ

\section{Elizabeth Kaluaratchige - Du désir au Nirvãna. Le destin bouddhique de la sexualité}

Résumé: Cet article veut prolonger jusqu'au bouddhisme, le dialogue que Freud a commencé autour de l'expérience religieuse du monothéisme. Sans appliquer la psychanalyse au bouddhisme, nous traitons le rapport entre le bouddhisme et la culture, pris par l'idéal «morale sexuelle civilisée des bouddhistes». Notre réflexion sur le thème «sexualité» dans le bouddhisme, tient compte de l'usage des concepts désignant non seulement les «comportements observables », mais les phénomènes de la vie qui restent à l'arrière plan inconscient.

Mots-clés : Bouddhisme - Sexualité - Désir - Plaisir - Nirvãna.

Elizabeth Kaluaratchige - From Desire to Nirvana. The Buddhist Destiny of Sexuality.

Abstract : This paper extends the discussion Freud initiated on the religious experience of monotheism into the sphere of Buddhism. While not attempting to offer a psychoanalytical reading of Buddhism, we shall examine the relationship between Buddhism and culture, from the vantage point of the ideal concerning the 'civilised sexual morality of Buddhists.' Our exploration of the theme of 'sexuality' in Buddhism uses concepts associated not only with 'observable behaviour', but also with phenomena in daily life rooted in the unconscious.

Key-words : Buddhism - Sexuality - Desire - Pleasure - Nirvana. 